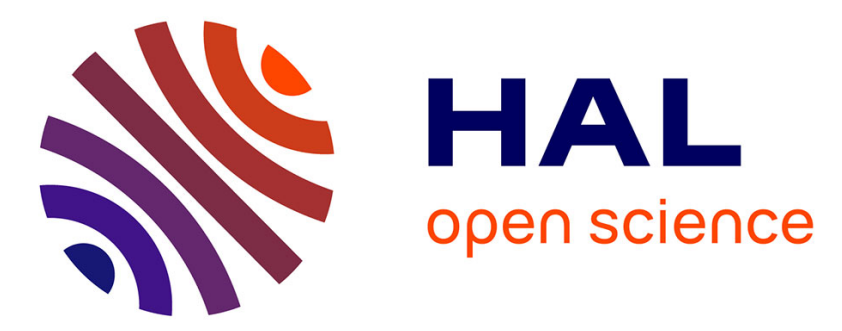

\title{
Direct Stress-Strain Measurements from Bulged Membranes Using Topography Image Correlation
}

Jan Neggers, Johan Hoenagels, François Hild, Stéphane Roux, Marc Geers

\section{To cite this version:}

Jan Neggers, Johan Hoenagels, François Hild, Stéphane Roux, Marc Geers. Direct Stress-Strain Measurements from Bulged Membranes Using Topography Image Correlation. Experimental Mechanics, 2014, 54 (5), pp.717-727. hal-01003511

\section{HAL Id: hal-01003511 https://hal.science/hal-01003511}

Submitted on 10 Jun 2014

HAL is a multi-disciplinary open access archive for the deposit and dissemination of scientific research documents, whether they are published or not. The documents may come from teaching and research institutions in France or abroad, or from public or private research centers.
L'archive ouverte pluridisciplinaire HAL, est destinée au dépôt et à la diffusion de documents scientifiques de niveau recherche, publiés ou non, émanant des établissements d'enseignement et de recherche français ou étrangers, des laboratoires publics ou privés. 


\title{
Direct stress-strain measurements from bulged membranes using topography image correlation
}

\author{
J. Neggers ${ }^{1}$ - J.P.M. Hoefnagels ${ }^{1} \cdot$ F. Hild ${ }^{2} \cdot$ S. Roux ${ }^{2}$ - M.G.D. Geers ${ }^{1}$
}

Received: 2 July 2013 / Accepted: 15 November 2013 / Published online: 9 January 2014

\begin{abstract}
This paper discusses an experimental method to characterize thin films as they are encountered in micro-electronic devices. The method enables the measurement of the stress and strain of pressure deflected bulged membranes without using a priori defined bulge equations. An enrichment to the Global Digital Image Correlation method is detailed to capture the membrane strain and curvature while robustly dealing with acquisition noise. The accuracy of the method is analyzed and compared to the standard bulge test method. The method is applied to a proof of principle experiment to investigate its applicability and accuracy. Additionally, it is shown for two experimental cases that the method provides accurate results, although the bulge equations do not hold.
\end{abstract}

Keywords digital image correlation · surface profilometry · thin film · membrane $\cdot$ full-field measurement · strain $\cdot$ stress

\section{Introduction}

Thin films are key in miniaturizing components in consumer electronic devices. It is known that the properties of most thin films are size dependent, since their intrinsic microstructural length scale is of comparable size to the design length scale $[1,2]$. Moreover, the processing techniques, and the adjacent materials, also influence the mechanical response $[3,4]$. It is therefore important to perform an in-situ characterization of these films,

1 Eindhoven University of Technology, Department of Mechanical Engineering, 5612 AZ Eindhoven, Netherlands Email: j.p.m.hoefnagels@tue.nl · 2 LMT Cachan, ENS Cachan / CNRS / UPMC / PRES UniverSud Paris

61 avenue du Président Wilson, 94235 Cachan Cedex, France i.e. with the selected manufacturing processes as used in the device.

The bulge-test is a well known technique for testing thin film properties, especially at small scales [1]. In a bulge-test, a membrane of specific shape (rectangular, circular) is deflected under pressure. The deflection and pressure can be converted into stress and strain using shape specific equations. Generally, bulge equations are derived using energy minimization methods [5-7] resorting to approximate membrane displacement fields. In these derivations several assumptions are used to make the system solvable. Although these assumptions may be clearly specified, they are often only approximately fulfilled and hence the resulting error/uncertainty on the estimates of the mechanical properties of these films is difficult to evaluate.

For micro-electronic systems the miniaturized rectangular bulge equations developed by Vlassak et al. [8, 9] are popular. The reason is that, for these systems, it is possible to manufacture the rectangular membranes with high precision by back etching a Si wafer using $\mathrm{KOH}$ (potassium hydroxide), thereby improving the accuracy significantly. Vlassak et al. have shown that for rectangular membranes (width $2 a$ along the $x$ direction, length $2 b$ along the $y$ axis, and thickness $t$ ), with sufficiently large width-to-length aspect ratio $b / a>6$, the center part of the membrane closely approximates a plane-strain state and deflects cylindrically. The bulge equations then obtained to compute the stress and strain 
from the pressure and deflection are,

$\kappa_{x x}=\frac{2 \delta}{a^{2}+\delta^{2}}$,

$\sigma_{x x}=\frac{P}{t \kappa_{x x}}$

$\varepsilon_{x x}=\frac{1}{a \kappa_{x x}} \sin ^{-1}\left(a \kappa_{x x}\right)-1$,

where, $P$ is the pressure and $\delta$ the deflection at the center (or apex) of the membrane and $\kappa_{x x}$ its curvature along direction $x$. Equation (2) is also known as the hoop stress equation, and is derived from static equilibrium assuming negligible flexural moment. Equation (3) is purely geometrical and based on the assumption that the membrane deflects to a circular profile. It has been shown that this method is accurate for films with large width-to-thickness ratios $a / t>1000$ (depending on the level of strain) because these thin films approximate a free rotating hinge at the boundary [20]. Thicker films conform poorly to the circular deflection profile that connects the two edges. In particular close to their edge, flexural moments are the largest and hence the evaluation of the curvature from Equation (1) may lead to significant errors. Alternatively, as long as the film edges are removed from the analysis, the hoop stress equation, Equation (2) may still be valid.

More importantly, all bulge equation methods are limited to homogeneous, unstructured membranes, with specific shapes. Nonetheless, in current microelectronic applications more complex thin film shapes are often found $[11,12]$, even with multiple layered and structured heterogeneous thickness materials. Since a bulgeequation based method can only provide a global (or averaged) material response, it would not apply to such thin films.

In this paper, a new method is proposed for measuring thin films that are loaded with a pressure medium similar to the bulge test method. In this new method, full-field Optical Confocal Profilometry is combined with dedicated Global Digital Image Correlation (GDIC). The proposed method enables the full-field strain and curvature fields to be measured for any type of deformed sample measured with a profilometric full-field technique.

Using the fact that the pressure is uniform under the sample, the full-field membrane stresses are derived from the pressure and curvature if the bending stresses are negligible and the local stress state (e.g., planestrain, biaxial loading) is known (Section 2.3). The proposed method utilizes the wealth of full-field data that allows for noise attenuation, resulting in improved accuracy in cases where the bulge equations apply, as discussed in Section 3. Moreover, the proposed method is direct, i.e. measuring the local stress and strain without assuming any global deformed shape. The method is therefore more widely applicable in terms of e.g. membrane thickness, membrane shapes, and structured membranes, as demonstrated in Section 4.

\section{Methods}

The proposed method has been developed for thin films as they are used in micro-electronics applications. For these films, the small strain regime is usually the most relevant for service conditions. This regime also implies small curvatures, which puts high demands on the used experimental technique. Therefore, a microscopic surface profilometry technique (such as confocal profilometry or phase shift interferometry) is chosen as a measurement method. This type of system measures the "surface profile" of the sample, which is the height of the sample at every pixel location. The resulting data are images (i.e. 2D matrices), where the pixel information is not a gray value but height. As a result, in this study, GDIC is applied to identify the 3D displacement vector field, from (2D) height-images, and therefore called Quasi-3D. Such a Quasi-3D GDIC method has been used before on Atomic Force Microscopy data [13]. Additionally, the proposed method aims to capture the curvature field, which is the second derivative of the position field. It is well known that noise has a detrimental effect on derivatives. It is therefore imperative to deal with the acquisition noise robustly, for which particular choices are made in the GDIC procedure.

\subsection{Global Digital Image Correlation}

Digital Image Correlation (DIC) in general consists of measuring the displacement field between two images obtained from two increments of loading in an experiment [14-16]. The first image is usually a picture of the reference configuration $f$, and the second image a picture of the deformed configuration $g$. If the pictures capture a (speckle) pattern, which is deformed with the underlying sample, then the gray level conservation for a pixel at location $\mathbf{x}$ reads

$\eta(\mathbf{x})=f(\mathbf{x})-g\left(\mathbf{x}+u(\mathbf{x}) \mathbf{e}_{x}+v(\mathbf{x}) \mathbf{e}_{y}\right)$,

where $u$ and $v$ are the in-plane components of the displacement respectively in $x$ and $y$ direction, and $\eta$ is the acquisition noise. In the case of profilometric data, the pixel values contain height information, which may evolve between two measurement increments. Thus the 
gray level conservation is rewritten as "topography" conservation,

$\eta(\mathbf{x})=f(\mathbf{x})-\left(g\left(\mathbf{x}+u(\mathbf{x}) \mathbf{e}_{x}+v(\mathbf{x}) \mathbf{e}_{y}\right)-w(\mathbf{x})\right)$,

where $w$ denotes the out-of-plane displacement, and $\eta$ now represents noise, but also height distortions not captured in $w$. Note that the topography is in data structure similar to a $2 \mathrm{D}$ image, thus the position vector remains $2 \mathrm{D}$,

$\mathbf{x}=x \mathbf{e}_{x}+y \mathbf{e}_{y}$,

in contrast with the $3 \mathrm{D}$ displacement vector,

$\mathbf{u}(\mathbf{x})=u(\mathbf{x}) \mathbf{e}_{x}+v(\mathbf{x}) \mathbf{e}_{y}+w(\mathbf{x}) \mathbf{e}_{z}$.

To write the problem in terms of a limited number of unknowns, the displacement field is obtained through the minimization of a functional over of subspace of functions $\varphi$,

$\mathbf{u}(\mathbf{x}) \approx \mathbf{u}^{*}(\mathbf{x}, \underline{\lambda})=\sum_{i=1}^{3 n} \lambda_{i} \varphi_{i}(\mathbf{x})$

where $\underline{\lambda}$ is a column vector with the degrees of freedom for a chosen set of basis functions $\varphi$, detailed later on. The displacement field $\mathbf{u}^{*}$ is then obtained by minimizing the squared residual with respect to $\underline{\lambda}$,

$\underline{\lambda}=\underset{\underline{\lambda}}{\operatorname{argmin}}(\Phi)=\underset{\underline{\lambda}}{\operatorname{argmin}} \int_{\Omega}(\eta(\mathbf{x}, \boldsymbol{\lambda}))^{2} \mathrm{~d} \mathbf{x}$,

where $\eta$ is now dependent on the degrees of freedom through the substitution of Equation (8) into Equation (5).

For this purpose, an iterative procedure is performed, where $\underline{\lambda}$ consists of a current value $\underline{\lambda}^{k}$, and an unknown iterative update $\underline{\delta \lambda}$

$\underline{\lambda}^{k+1}=\underline{\lambda}^{k}+\underline{\delta \lambda}$.

where $k$ denotes the current iteration. The update is found by solving the linearized form of Equation (9),

$\forall_{j} \sum_{i=1}^{3 n} \int_{\Omega}\left(\boldsymbol{\varphi}_{j} \cdot \mathbf{G}\right)\left(\mathbf{G} \cdot \boldsymbol{\varphi}_{i}\right) \mathrm{d} \mathbf{x} \delta \lambda_{i}=\int_{\Omega} \boldsymbol{\varphi}_{j} \cdot \mathbf{G}(f-\tilde{g}) \mathrm{d} \mathbf{x}$,

where $\mathbf{G}$ is the image gradient, defined below, and,

$\tilde{g}=g\left(\mathbf{x}+u^{*}\left(\mathbf{x}, \underline{\lambda}^{k}\right) \mathbf{e}_{x}+v^{*}\left(\mathbf{x}, \underline{\lambda}^{k}\right) \mathbf{e}_{y}\right)-w^{*}\left(\mathbf{x}, \underline{\lambda}^{k}\right)$,

is the corrected version of $g(\mathbf{x})$ using the current estimate of the displacement field. Equation (11) is usually written in matrix form,

$\underline{\underline{M}} \underline{\delta \lambda}=\underline{b}$, where the tangent matrix $\underline{\underline{M}}$ and the right-hand member $\underline{b}$ are conceptually similar to established GDIC [16, 17]. The main difference is the vector $\mathbf{G}$, which is the image gradient enriched with an out-of-plane component

$\mathbf{G}=\frac{\partial f}{\partial x} \mathbf{e}_{x}+\frac{\partial f}{\partial y} \mathbf{e}_{y}-\mathbf{e}_{z}$,

and the three-dimensional nature of the basis functions

$\boldsymbol{\varphi}=\varphi_{x} \mathbf{e}_{x}+\varphi_{y} \mathbf{e}_{y}+\varphi_{z} \mathbf{e}_{z}$

To build $\tilde{g}(\mathbf{x})$, the image $g(\mathbf{x})$ needs to be estimated at locations $\mathbf{x}+\mathbf{u}_{2}(\mathbf{x})$, for which a cubic spline interpolation scheme is used, where $\mathbf{u}_{2}$ denotes the in-plane displacement field. This interpolation is not exact, and is a source of error $[18,19]$. Interestingly, the out-of-plane component does not enter the interpolation scheme, and does not suffer from interpolation errors in a direct way.

The iterative system is solved until convergence is reached. The chosen criterion is such that the norm of the degree of freedom increments, $\underline{\delta \lambda}$, becomes sufficiently small, i.e. $\|\underline{\delta \lambda}\|<10^{-6}$.

The implementation used in this paper is considered a "global" DIC method because the system is minimized globally, instead of minimizing each zone of interest independently. However, the out-of-plane enrichment can also be applied to "local" DIC methods by following exactly the same procedure.

\subsection{Basis Functions}

In a general DIC problem, a displacement vector is to be determined for each pixel in an image. This makes the problem ill-posed, even when not considering image noise. The solution is to reduce the number of unknowns to less than the number of pixels, for instance as was done in Equation (8). If there is uncorrelated (i.e. white) noise, then using more pixels per unknown will further reduce the noise sensitivity, thereby improving the accuracy. However, using too few unknowns restricts the kinematics of the solution, which decreases the accuracy. For that reason, it is important to use a basis that adequately captures the full kinematics of clamped bending membranes, inside the Region Of Interest (ROI), with a minimum number of degrees of freedom. Inspired by continuum solutions for loaded plates, a $2 \mathrm{D}$ polynomial basis is used. The $C^{\infty}$ continuity property of polynomials makes these functions well suited for calculating curvature fields, noted that polynomials of at least second order are required to be sensitive to curvature. 

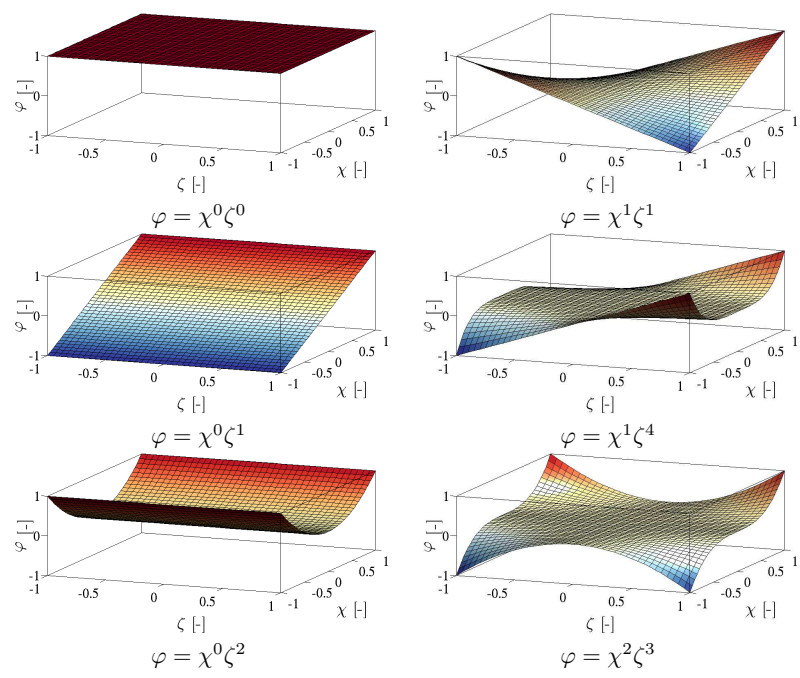

Fig. 1 Example 2D basis-functions $\varphi$.

The basis functions are vector functions where one component of the field is expressed as the product of two terms of a polynomial series,

$\varphi_{x}=\varphi_{y}=\varphi_{z}=\chi^{a} \zeta^{b}$,

where $\chi$ and $\zeta$ are normalized coordinates for $x$ and $y$ such that $-1 \leq \chi, \zeta \leq 1$. The complete basis is formed by using all combinations of $a$ and $b$ up to a certain order $p$, see Figure 1 for some examples. Each basis function is used three times, each with its own degree-of-freedom (for each component of the displacement vector). For example for $p=2$,

$$
\begin{aligned}
u^{*} & =\lambda_{1} \chi^{0} \zeta^{0}+\lambda_{2} \chi^{0} \zeta^{1}+\lambda_{3} \chi^{1} \zeta^{0} \\
& +\lambda_{4} \chi^{0} \zeta^{2}+\lambda_{5} \chi^{1} \zeta^{1}+\lambda_{6} \chi^{2} \zeta^{0}, \\
v^{*} & =\lambda_{7} \chi^{0} \zeta^{0}+\lambda_{8} \chi^{0} \zeta^{1}+\lambda_{9} \chi^{1} \zeta^{0} \\
& +\lambda_{10} \chi^{0} \zeta^{2}+\lambda_{11} \chi^{1} \zeta^{1}+\lambda_{12} \chi^{2} \zeta^{0}, \\
w^{*} & =\lambda_{13} \chi^{0} \zeta^{0}+\lambda_{14} \chi^{0} \zeta^{1}+\lambda_{15} \chi^{1} \zeta^{0} \\
& +\lambda_{16} \chi^{0} \zeta^{2}+\lambda_{17} \chi^{1} \zeta^{1}+\lambda_{18} \chi^{2} \zeta^{0} .
\end{aligned}
$$

This particular decomposition into separate degrees of freedom for each component of the displacement vector is one of the possible ways to define the basis functions. In particular, this will give 3 basis functions for $p=0$, 9 basis functions for $p=1,18$ basis functions for $p=2$, 30 basis functions for $p=3$, etc.

Because of the fact that the matrix elements $M_{i j}$ involve the scalar product of pairs of functions with a "weight" that is the tensor $G \otimes G$, there is no gain in choosing an orthogonal polynomial basis. The same space of function will be generated by the above polynomials and say Legendre polynomials of the same order. Hence the resulting displacement, and convergence rate is independent of the particular choice provided the generated space is the same.

\subsection{Curvature, Stress and Strain}

Let us revisit the assumptions in the bulge equations discussed in the introduction. Equation (1) is a purely geometrical formulation to obtain the curvature from the deflection, assuming the membrane deflects according to a circular profile. For thin membranes and deflections larger than the membrane thickness this assumption holds, attributing to the established accuracy of the bulge test method. However, with the GDIC method discussed in this paper the curvature tensor $\boldsymbol{\kappa}(\mathbf{x})$ at each pixel location can be computed without resorting to the circularity assumption (see also [20]), by first defining

$\kappa(\mathbf{x})=\nabla \mathbf{n}(\mathbf{x})$,

where $\mathbf{n}(\mathbf{x})$ is the normal vector, which in turn is the gradient of the position field $z(\mathbf{x})$ (corrected for rotations)

$\mathbf{n}(\mathbf{x})=\frac{\nabla z(\mathbf{x})}{\|\nabla z(\mathbf{x})\|}$.

The position field is measured as the topography $g(\mathbf{x})$, however, it also includes the additional pattern. The membrane topography (i.e. the required position field) is more accurately obtained by by applying the measured displacement field to the initial (flat) membrane position. Because the displacement fields are forced to be smooth, by the polynomial basis, noise in the curvature is here controlled by the basis order, as will be shown further down.

Equation (3) is a similar geometrical formulation as Equation (1), which expresses the strain as function of the curvature assuming a circular deflection profile. For thicker or inhomogeneous membranes the circular deflection profile assumptions is violated, especially at the membrane boundary and heterogeneous points. However, the GDIC method recovers the displacement field, from which the strain field is easily obtained without assumptions, by e.g. computing the Green-Lagrange strain tensor E in the membrane plane.

Equation (2) is a simple balance equation evaluated without recourse to the small displacement hypothesis. It is valid as long as the bending moment can be neglected, but the actual size of the membrane does not come into play. Its validity depends on the flexural moment within the region over which the curvature is evaluated. However, the flexural moment is proportional to the change in curvature, which is measured and thus can be assessed.

At the apex of bulged square membranes, the pressure is balanced with membrane stresses in two directions. If the curvatures in both directions are equal then 


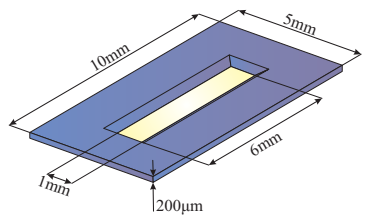

(a)

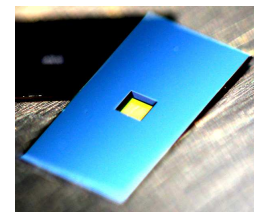

(b)
Fig. 2 The two sample types used in the experiments, the $\mathrm{Si}_{3} \mathrm{~N}_{4}$ membrane is $100 \mathrm{~nm}$ thick in both types, (a) a drawing of a rectangular membrane $\left(1 \times 6 \mathrm{~mm}^{2}\right)$, (b) a photograph of a square membrane $\left(1 \times 1 \mathrm{~mm}^{2}\right)$.

the principal stresses are also equal if the material is isotropic,

$\sigma_{x x}=\sigma_{y y}=\frac{P}{2 t \kappa}$.

If a part of the membrane deforms axisymmetrically, the principal stresses of that membrane part are related to the meridional and circumferential curvatures [21, $22]$,

$\sigma_{m}=\frac{P}{2 t \kappa_{c}}$,

$\sigma_{m}=\frac{P}{t \kappa_{c}}\left(1-\frac{\kappa_{m}}{2 \kappa_{c}}\right)$.

where $c$ and $m$ denote the meridional and circumferential directions respectively. For kinematically more complex cases, closed-form expressions cannot be obtained, and numerical schemes are required, such as Finite Element Method Updating [23].

To conclude, the assumption of neglecting flexural stiffness remains in the proposed methodology, yet only for relating stress to curvature. The assumption is appropriate if a restrained region is analyzed, such that the change in curvature is insignificant over the analyzed region.

\subsection{Experimental Procedure}

Two types of samples are used in the present paper, a square $\left(1 \times 1 \mathrm{~mm}^{2}\right)$ membrane and a rectangular $\left(1 \times 6 \mathrm{~mm}^{2}\right)$ membrane (Figure 2). Both samples are manufactured by deposition a $100 \mathrm{~nm}$ thick $\mathrm{Si}_{3} \mathrm{~N}_{4}$ layer on a monocrystalline $200 \mu \mathrm{m}$ thick Si wafer. A freestanding membrane is created by back $\mathrm{KOH}$ etching the wafer up to the $\mathrm{Si}_{3} \mathrm{~N}_{4}$ layer. The membrane dimensions are chosen such that the bulge equations are known to perform well [8], enabling a comparison with the GDIC method presented in this paper.

These samples are processed with high precision micromanufacturing techniques, resulting in a surface roughness that is smaller than measurable with the used optical profilometer. Since a height correlation is to be

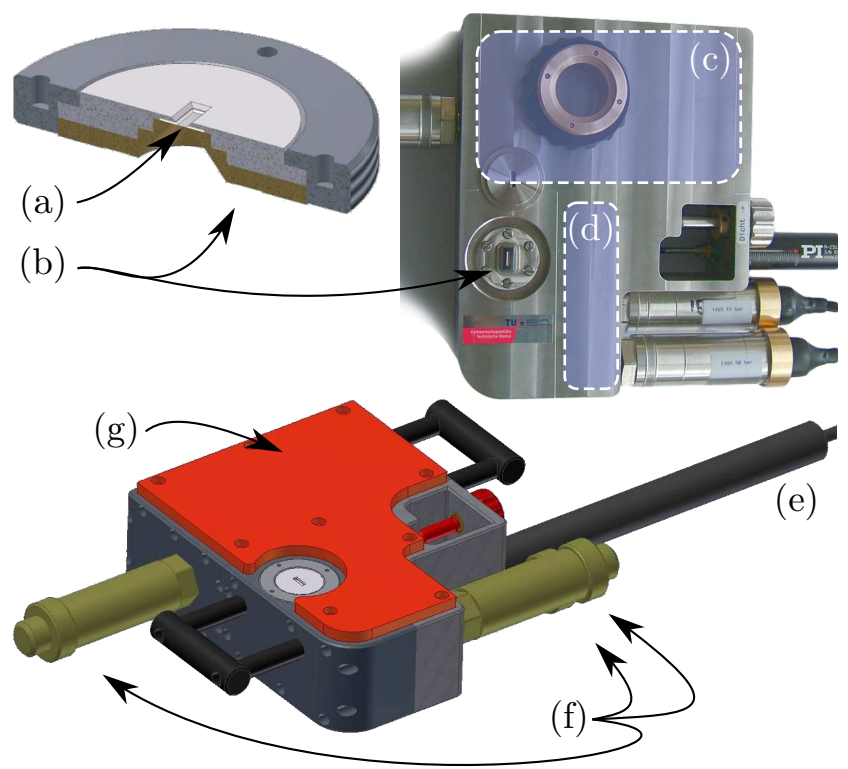

Fig. 3 The bulge test apparatus with, (a) the sample, (b) the sample holder, (c) the $270 \mathrm{ml}$ chamber, (d) the $30 \mathrm{ml}$ chamber, (e) the linear actuator connected to the piston, (f) the three pressure sensors, (g) the heating stage.

performed on these samples, a height pattern is required. This pattern is created by coating the membrane surface with a colloidal suspension of Ag particles (80-500 nm diameter) dispersed in ethanol. After evaporation of the suspending fluid, the deposited particles (or rather clusters of particles as they tend to aggregate) showed enough physical adhesion to withstand the bulge test without detaching from nor moving on the surface. Note that the basis functions in the GDIC method have support over the entire ROI, allowing the GDIC method to work efficiently with relatively sparse patterns. The sparse pattern allowed the evaluation of the unloaded freestanding film with and without the pattern. Application of the pattern did not cause a measurable deformation, confirming the insignificant influence on the mechanics.

To test the membranes a custom made low profile bulge test apparatus is specifically designed to allow for optical access to the sample surface (Figure 3). The apparatus consists of two ethanol filled stainless steel chambers of $270 \mathrm{ml}$ and $30 \mathrm{ml}$ respectively (Figure $3 \mathrm{~cd}$ ), which are connected with a valve. The sample is mounted on the smaller chamber with an interchangeable sample holder (Figure 3b). Additionally, the apparatus is equipped with three pressure sensors with respective pressure ranges $[0-5],[0-15],[0-50]$ bar (Figure 3f). The pressure sensors are high performance millivolt output transducers from GE Sensing, with an accuracy of $0.04 \%$ of their rated maximum. The two large range sensors are mounted on the smaller cham- 
ber while the most sensitive sensor is mounted on the larger chamber. The pressure difference is applied with a piston radius of $1.6 \mathrm{~mm}$, connected to the smallest chamber, driven by a linear actuator with a closed-loop DC motor (Figure 3e). The actuator steps in $50 \mathrm{~nm}$ increments with a total stroke of $25 \mathrm{~mm}$, and a maximum applied force of $70 \mathrm{~N}$. The two chamber design allows experiments in two pressure ranges, i.e. by closing the valve to the second chamber the most sensitive pressure sensor is naturally protected from overloading, while at the same time, the effect of the piston motion on the pressure is amplified. Finally, a heating stage (Figure 3g) serves to keep the entire system at a constant temperature, slightly above the room temperature $\left(\sim 28^{\circ} \mathrm{C}\right)$, to eliminate pressure changes due to thermal expansion.

The bulge test apparatus fits under the Sensofar Optical Profilometer, which is used to measure the surface roughness of each pressure increment. The experimental procedure is controlled via custom NI LabVIEW code that follows a user-defined pressure loading curve in a closed loop, with intermittent pressure hold periods for the profile measurements. The height profile is captured by a Sensofar Pl 12300 Optical Confocal Microscope using a CCD camera (definition: $557 \times 557$ pixels) with a Nikon EPI 50x objective lens, resulting in a square field of view of $184 \times 184 \mathrm{\mu m}^{2}$. The objective lens has a numerical aperture of 0.80 , which together with the monochromatic blue light $(\lambda=470 \mathrm{~nm})$ results in an in-plane resolution of $358 \mathrm{~nm}$ (i.e. slightly larger than the resolution of one pixel of the CCD sensor (332 nm)). For the patterned membranes discussed in this paper, the out-of-plane resolution has been measured to be $\sim 20 \mathrm{~nm}$ from the RMS of the difference between two height profiles of the same area on the sample. The difference between in-plane and out-of-plane resolution will also be apparent in the accuracy analysis of Section 3.

\subsection{Virtual Experiment}

In this paper, proof of principle experiments on square and rectangular membranes are performed. To make a thorough evaluation of the accuracy of the method, virtual experiments are used. A virtual experiment is a synthetic procedure that produces similar data as expected from the real experiment, yet, with the additional knowledge of the reference.

To create realistic virtual height profiles, the virtual experimental procedure consists of deforming a real experimental height profile $f$ (Figure $4 \mathrm{~b}$ ) with an FE displacement field (Figure 4c) to obtain a virtual deformed height profile $g$ (Figure 4d). Different increments in the

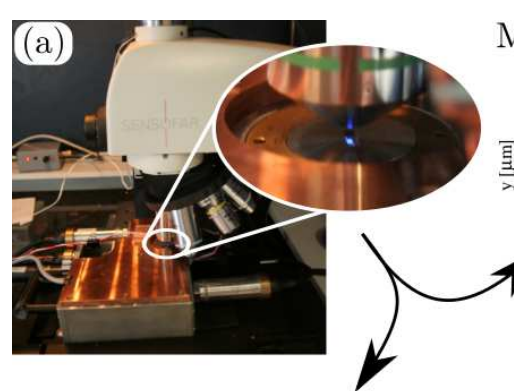

Measured Profile $g(\mathbf{x})$

Measured Profile $f(\mathbf{x})$

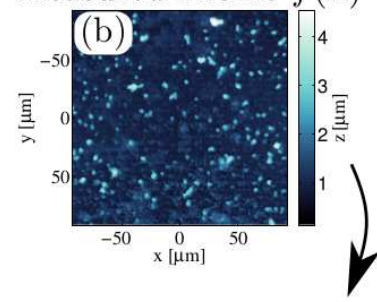

Virtual Profile $g(\mathbf{x})$

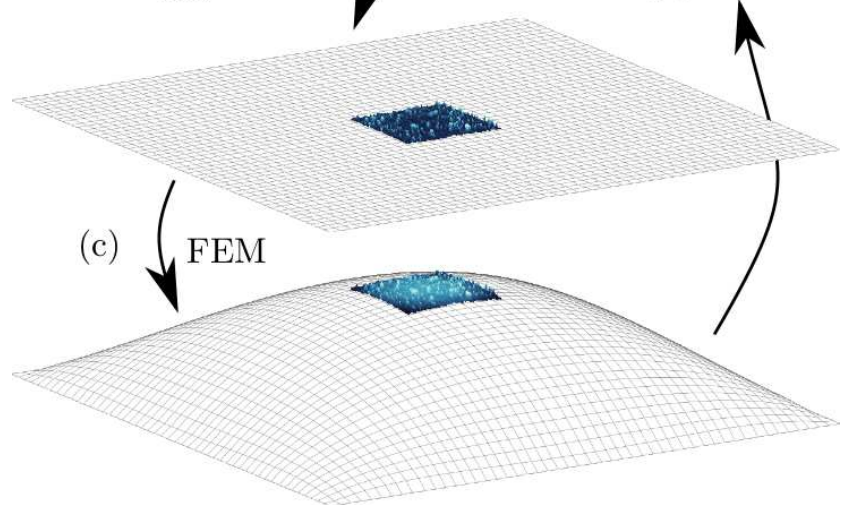

Fig. 4 The reference profile $f(\mathbf{x})$ and the deformed profile $g(\mathbf{x})$ are measured with the experimental setup (a,b,e). Additionally, the reference profile is virtually deformed with an FE displacement field (c) to obtain a virtually deformed profile (d), which approximates the real experiment.

FE simulation are used to obtain the incremental pressure height profiles similar to a real experiment.

Two different FE geometries are used, corresponding to the rectangular (Figure 2a) and to the square (Figure 2b) membrane. In the FE simulations, a dense mesh is chosen to match the pixel discretization level of the height images. This results in a mesh of approximately 50,000 3D 4-node bilinear Mindlin shell elements, which is densest at the field of view (FOV) and opens towards the boundaries. Note that, for illustrative reasons, the grid shown in (Figure 4c) is not the actual used mesh, which would be too dense to show any meaningful detail.

At this stage it is possible to determine the order of the basis functions required to accurately capture the kinematics in the virtual experiment. Figure 5 shows the mean absolute displacement error between the FE displacement field $\mathbf{u}_{\text {fem }}$ and displacement fields approximated with increasing polynomial order $\mathbf{u}^{*}$. An error measure is introduced as the average of the lengths 


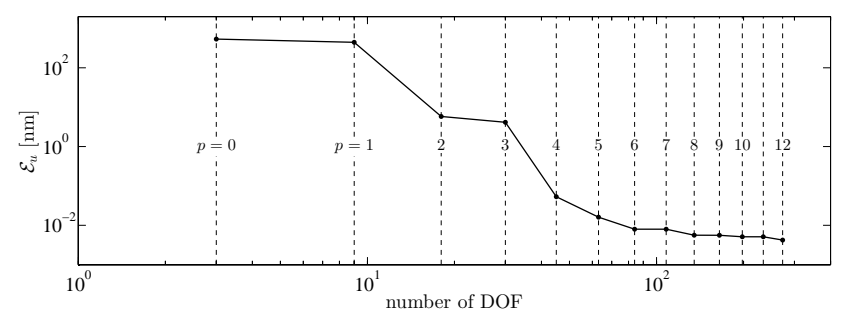

Fig. 5 The mean absolute error as a function of polynomial order obtained by comparing the FE displacement field with approximated displacement fields with increasing polynomial order.

of all difference vectors between the FE displacement field and the approximated displacement fields, evaluated over all pixels $k$,

$\mathcal{E}_{u}=\frac{1}{n} \sum_{k=1}^{n}\left(\left\|\mathbf{u}_{\mathrm{fem}}\left(\mathbf{x}_{k}\right)-\mathbf{u}^{*}\left(\mathbf{x}_{k}\right)\right\|\right)$

where the norm is defined as the Euclidean norm of, in this case, the difference vector. As expected, the mean absolute error decays for increasing order, and reaches an error level of less than one nanometer for sets of basis functions with $p \geq 4$. Since this error is smaller than the resolution of the measurement system, it is chosen to truncate the series of basis functions at $p=4$.

\section{Accuracy Analysis}

\subsection{Error Fields}

The use of the virtual experiment allows for direct comparisons between the results obtained with the proposed GDIC method and the FE reference solution. Similarly to Equation (21), a pixel-wise displacement error is computed as the vector difference between reference and the displacements estimated by GDIC. The different components and magnitude of the displacement error fields are shown in Figure 6 for the correlated ROI. More importantly, Figure 6a shows that the maximum error in displacement is approximately $10 \mathrm{~nm}$ while the average absolute error is much smaller. Even though there is no noise present in this virtual experiment, the result is based on a real experimental image $f$ with the same pixel discretization and pattern as the real experiment. Note the difference in the in-plane accuracy versus the out-of-plane accuracy (Figure 6b-d). The error in $w$ is approximately 20 times smaller than in $u$ and $v$. This coincides with the observed accuracy of the confocal microscopy set-up itself.

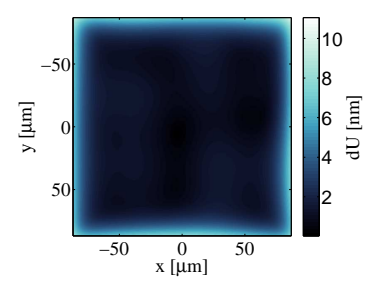

(a) Norm of the displacement error field

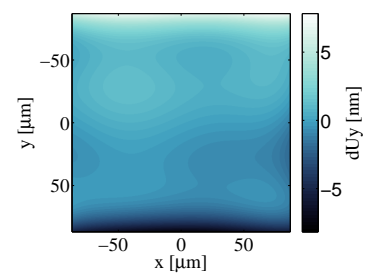

(c) $y$-component of the displacement error field

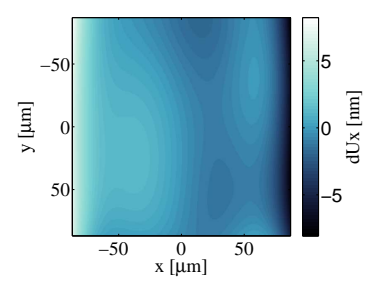

(b) $x$-component of the displacement error field

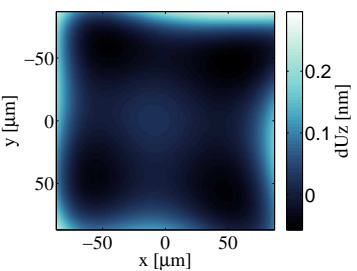

(d) z-component of the displacement error field
Fig. 6 Error in the estimated displacement fields for the ROI for a full $4^{\text {th }}$ order set of shape functions without additional noise. The Euclidean norm is used as the vector norm shown in subfigure (a). Note that the accuracy of the out-of-plane displacement is approximately 20 times better than the accuracy of the in-plane displacement, which coincides with the observed accuracy of the confocal microscopy set-up.

\subsection{Error vs. number of DOFs}

The error sources in DIC are in general threefold:

1. acquisition noise or pattern changes;

2. subpixel interpolation;

3. displacement approximation.

To minimize the impact of the second error sources, a local cubic spline subpixel interpolation method is used. More interestingly, the first and third error source are dependent on the chosen basis. Using too few (or incorrect) basis functions will introduce systematic errors because the kinematics of the experiment cannot be captured. Conversely, giving the system more freedom, allows the solution to be sensitive to noise, resulting in statistical errors.

In Figures 7ab, image correlations are performed on virtual experiments for an increasing number of degrees of freedom, for various levels of (white) noise added to the deformed profile $g$. The virtual experiment method allows the application of artificial acquisition noise by adding generated noise fields to the images. The various noise levels are quantified as the standard deviation of the white noise, relative to the image gray level dynamics. It is clearly shown that for the noise-free situation, increasing the number of degrees of freedom only improves the result, while when noise is included, an optimum accuracy is found using a conservative number of 


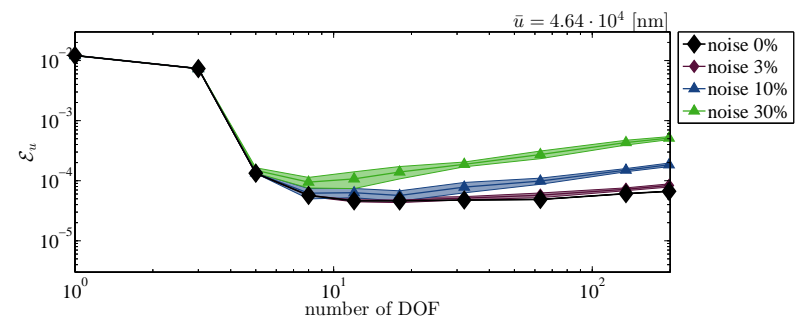

(a) Displacement error, normalized with the mean displacement $\bar{u}$

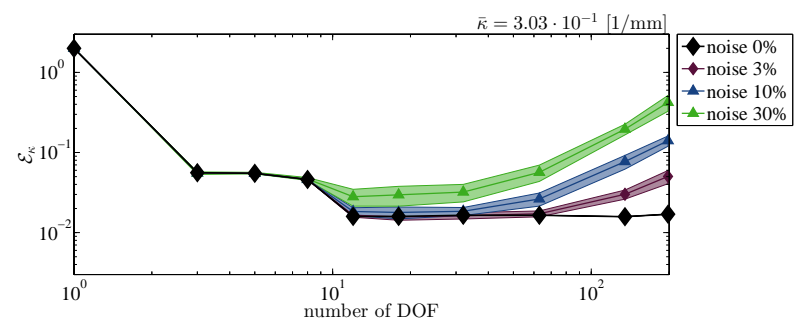

(b) Curvature error, normalized with the mean curvature $\bar{\kappa}$

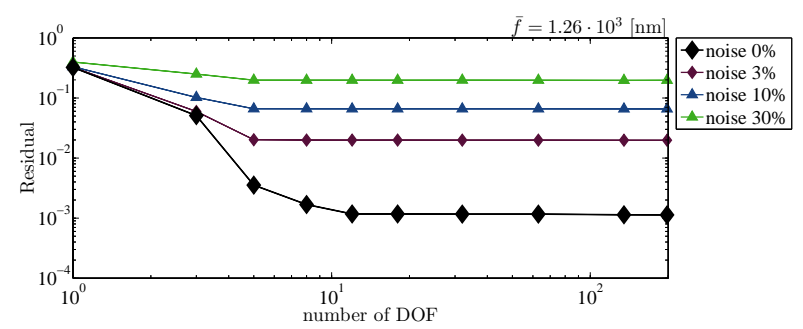

(c) Residual, normalized with the pattern RMS $\bar{f}$

Fig. 7 Effect of increasing the number of degrees of freedom on (b) the error in displacement, (c) the error in curvature, (a) the residual.

degrees of freedom (i.e. 10 to 20 DOFs). These results demonstrate that choosing the right kinematic basis for the GDIC procedure enhances the accuracy. The preferred basis is the one that captures the full kinematics with the minimum number of degrees of freedom. The applicability of a narrow basis, which is ideal in terms of accuracy and robustness, is limited to experiments within the range of this narrow basis. In contrast, the polynomial basis, discussed in this paper, is applicable to many types of experiments, provided they have slowly varying displacement fields, making the proposed method relatively general.

In a real experiment, no reference case exists. Therefore, the recommended procedure to select a proper set of basis functions, is to perform the correlation for increasingly rich sets of basis functions. The residual (Figure $7 \mathrm{c}$ ) will decrease for richer bases (if converged), yet the decrease will flatten at some level of richness. At this point, adding more degrees of freedom to the system tends to make the correlation less accurate due to noise sensitivity. Another method for evaluating the chosen basis, is by examining the residual field. A correlation with too few basis functions will result in a residual field exhibiting long wavelength modulations, while the residual field for an appropriate basis will only contain the acquisition noise, and thus will resemble white noise.

\section{Demonstration experiments}

\subsection{Proof of Principle Experiments}

To investigate the accuracy of the method on real cases, four experiments are performed: two experiments deal with rectangular membranes, and two with square ones. The samples are chosen for allowing optimal accuracy when using the bulge equations, to make a fair comparison with the discussed GDIC method, see Section 2.4.

The membranes are pressurized with the bulge test apparatus to a pressure of 1 bar in 100 increments (above atmosphere), and then unloaded to 0 bar in 20 increments. One increment takes approximately $90 \mathrm{sec}-$ onds including the pressure change, and scanning of the confocal microscope. On each of the recorded images the polynomial GDIC procedure is performed with all basis functions up to $p=2$ for the square membranes (i.e. 18 DOFs). For the rectangular membrane the DOFs that operate in $y$-direction are removed leaving 15 DOFs.

Figure 8 shows the stress and strain results after processing the topographies with both the GDIC method and the bulge equations. The square membrane response is more stiff, which is expected since it is in a more confined biaxial strain state when compared to the plane strain state at which the rectangular membrane is deformed. Consequently, the obtained moduli are the plane strain modulus and the biaxial modulus that are directly related to the Young's modulus $(E \approx 234 \mathrm{GPa})$ and the Poisson's ratio, $(\nu \approx 0.16)$ and are as expected for these thin $\mathrm{Si}_{3} \mathrm{~N}_{4}$ films. For the square membranes the stress-strain curves in both $x$ and $y$-directions are shown, using $\kappa_{x x}$ and $\kappa_{y y}$ respectively. The curves overlap showing that the membranes are not anisotropic, at least not within the membrane plane. More importantly, the figure shows that both the bulge equations and the GDIC method give the same results, confirming the validity of the method.

\subsection{Beyond the bulge equations}

The previous results show that the method is comparable to the accuracy obtained by using the bulge equations. However, the goal of this method is to go be- 


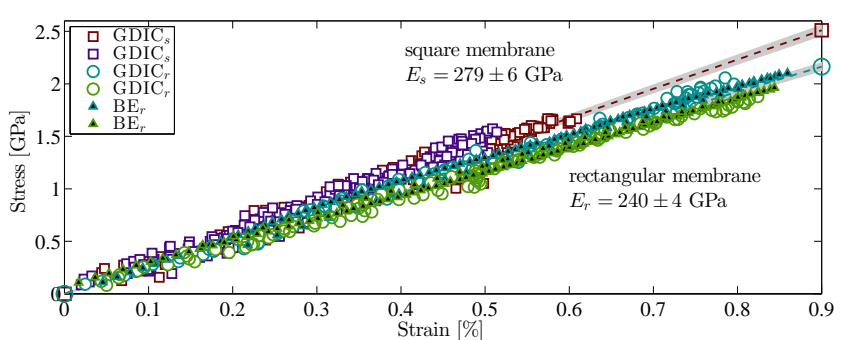

Fig. 8 Measured stress strain response for two square and two rectangular $100 \mathrm{~nm}$ thick membranes. The GDIC method and the bulge equations perform equally well for such thin membranes.

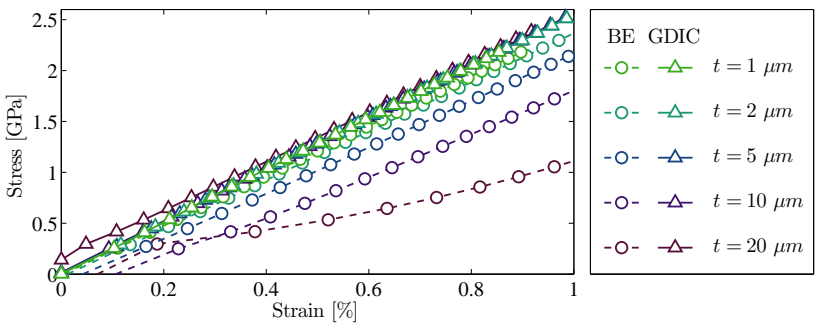

Fig. 9 GDIC method and the bulge equations applied to virtual experimental data with the same material properties, but with various membrane thicknesses. The GDIC results do not deviate from the reference elastic response up to a thickness of $20 \mu \mathrm{m}$.

yond the validity regime of the bulge equations. Therefore, the GDIC method is applied to data of thicker membranes which are created by the virtual experiment framework.

A number of virtual experiments are performed (see Section 2.5). All virtual experiments use the same linear elastic material properties with Young's modulus of $235 \mathrm{GPa}$. Figure 9 shows the stress strain response taken from the center of membranes for all virtual experiments. As expected the bulge equations start deviating from the correct response for thicknesses above $2 \mu \mathrm{m}$, for this $1 \mathrm{~mm}$ wide membrane. In contrast, applying the GDIC method to the same data shows correct stress strain response up to thicknesses of $20 \mu \mathrm{m}$. The GDIC method considers only the membrane inside the ROI, and thus is much less restricted by the flexural stiffness assumption.

For even thicker membranes the stress across the thickness is far from uniform, and no longer satisfies the assumptions made in Equation (2). It may be possible to obtain a corrected stress from the curvature for thicker membranes, but this requires a modification that is outside the scope of this paper.
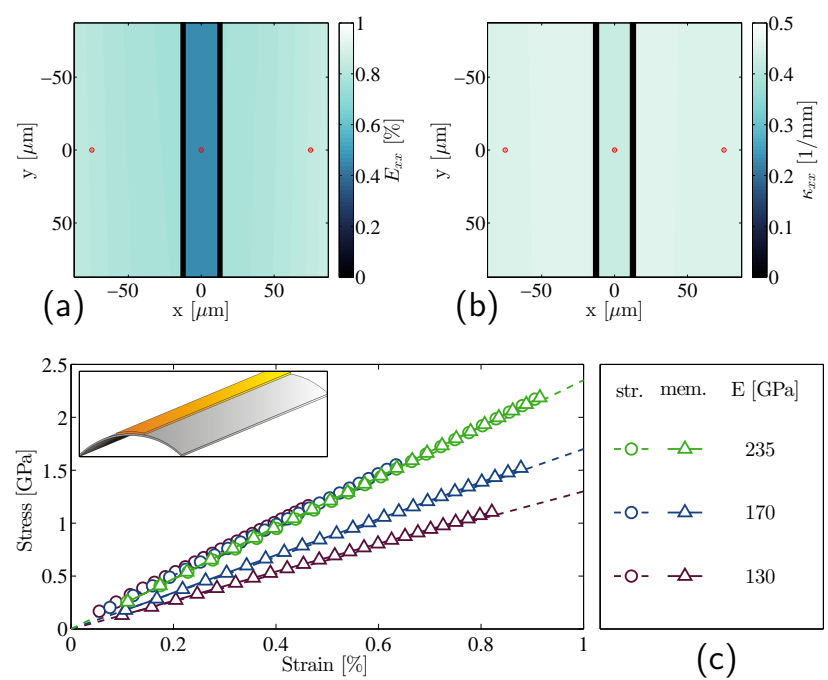

Fig. 10 GDIC applied to three heterogeneous membranes, where only the Young's modulus of the membrane material is varied, and the center strip of material has a constant modulus of $235 \mathrm{GPa}$. Note that the method can capture full-field strain (a) and curvature (b) fields. The red dots in (a,b) show the location where the stress-strain depicted in (c) is taken.

\subsection{Inhomogeneous Films}

The previous results use only the stress and strain at the center of the membrane, but the GDIC method gives full-field stress strain results. To show that the GDIC method captures features in the membranes, the virtual experiment framework is again applied to generate experimental data. In this case a $24 \mu \mathrm{m}$ wide strip along the y-axis is modeled using a Young's modulus of $E_{\text {str. }}=235 \mathrm{GPa}$, while the remaining membrane material is modeled three times with different moduli, i.e. $E_{\text {mem. }}=[130,170,235] \mathrm{GPa}$.

The generated topography images are processed with the GDIC method twice, once masking the structure, and once masking everything but the structure. For all three virtual experiments the stress-strain curves are collected in one point on the structure and in two points on the membrane (See Figure 10). This shows that it is possible to obtain the correct stress strain results for two different materials from a single full-field measurement.

With the proposed method it is always possible to obtain curvature fields and strain fields. However, obtaining the stress fields relies on applying either Equation (2), (18), or (19), which are only valid for particular cases (e.g. plane-strain, biaxial, or axisymmetric states). 


\section{Discussion and Conclusions}

The global DIC method has been extended to images containing height profiles, relaxing the brightness conservation principle and enabling for quantitative measurement of in-plane and out-of-plane displacements. This extension is useful for any topographic measurement, in particular optical confocal profilometry, but also e.g. Scanning Probe Microscopes like Atomic Force Microscopy. Furthermore, the out-of-plane quantity is not limited to topography, it can be any measured field, e.g., temperature [24].

In the proposed GDIC method the image pattern and the quantified out-of-plane displacement field are both taken from the same topographical data. In order to avoid interference between them, a separation in length scales is required between the length scale of the out-of-plane displacement fluctuations and the pattern length scale. For the presented case of bulged membranes this separation of length scales is achieved by applying low order polynomial basis functions with support over the entire region of interest and by controlling the surface marking with a deposition of small clusters of nanoparticles. Considering that the membrane mechanics are the goal of the analysis, it is important that the added pattern does not mechanically influence the membrane. For the discussed cases it was shown that the pattern did not significantly influence the mechanics.

The introduced polynomial basis is particularly suited for the bulging of membranes, and not limited to square or rectangular membranes. The polynomial basis functions have a wide support and capture the membrane kinematics with only a few degrees of freedom. This makes the method highly robust with respect to noise, but also with respect to the initial guess. Moreover, the measured displacement fields are $C^{\infty}$ continuous, making them ideal for the calculation of curvature fields.

Through the use of virtual experiments, where the input (FE) displacement field is known, the reference solution can be compared with the measured (via GDIC) displacement fields, through which the accuracy of the method was analyzed. The accuracy depends on the level of the acquisition noise and the richness of the kinematics that is controlled through the number of basis functions. Too restricted kinematics will give inaccurate results because the kinematics of the experiment is not adequately captured; too rich kinematics will make the solution sensitive to noise also leading to inaccurate results.

In a real experiment no reference displacement field exists to asses if an optimal basis is applied. To identify this optimum, it is suggested to perform DIC calcula- tions for increasing number of degrees of freedom until the residual field stabilizes. The residual field should always decrease for increasing number of degrees of freedom, but when the residual decrease becomes small, it suggests that modes that cannot be measured are progressively included thereby inducing a degradation of the conditioning of the tangent GDIC problem, and hence an increased noise sensitivity.

The proposed method recovers the displacement fields, from which strain fields are trivially computed, regardless of the shape or thickness of the membrane. Moreover, the proposed method recovers curvature fields, and it is shown that for particular cases (i.e. planestrain, biaxial, or axisymmetric strain-states) the stress fields can be recovered. Consequently, the bulge membrane shape can be any shape, and as long as the interrogated area fulfills one of these particular strain-states, then it is possible to obtain the stress in that respective area. For cases with more complex kinematics, a coupling with numerical method is to be made in order to obtain the stress.

As a proof-of-principle, bulge test experiments are performed for $100 \mathrm{~nm}$ thick Silicon Nitride films. Both the GDIC method and the plane-strain bulge equations are applied to the data, and give comparable results. This shows that the GDIC method at least matches the accuracy of the bulge equations for this particular case. However, the applicability regime of the GDIC method is much less restrictive.

The method applies the same assumption (negligible flexural moments) as the plane-strain bulge equations to obtain stresses. However, by considering only a small portion of the membrane, the assumption is less restrictive. Therefore, application of the method to inhomogeneous membranes with less restrictions on the membrane thickness has shown to accurately recover the stress-strain response of the various materials in the field of view. Consequently, the proposed method is less restricted in terms of membrane thickness, membrane homogeneity and membrane shapes, thereby, alleviating these shortcomings of the original bulge test.

\section{Acknowledgment}

This work has been supported by the Dutch Technology Foundation (STW) and the Dutch Organization for Scientific Research (NWO).

\section{References}

1. W. Nix, "Mechanical properties of thin films," Metallurgical transactions A 20A, 2217-2245 (1989). 
2. R. Vinci and J. Vlassak, "Mechanical behavior of thin films," Annual Review of Materials Science 26, 431-462 (1996).

3. E. Arzt, "Size effects in materials due to microstructural and dimensional constraints: a comparative review," Acta Materialia 46, 5611-5626 (1998).

4. P. Gruber, J. Böhm, F. Onuseit, A. Wanner, R. Spolenak, and E. Arzt, "Size effects on yield strength and strain hardening for ultra-thin $\mathrm{Cu}$ films with and without passivation: A study by synchrotron and bulge test techniques," Acta Materialia 56, 2318-2335 (2008).

5. J. Beams, Mechanical properties of thin films of gold and silver (John Wiley and Sons, New York, NY, 1959).

6. O. Tabata, K. Kawahata, S. Sugiyama, and I. Igarashi, "Mechanical property measurements of thin films using load-deflection of composite rectangular membranes," Sensors and Actuators 20, 135141 (1989).

7. S. Timoshenko and S. Woinowsky-Krieger, Theory of plates and shells (McGraw-Hill, New York, NY, 1987).

8. J. Vlassak and W. Nix, "A new bulge test technique for the determination of Young's modulus and Poisson's ratio of thin films," Journal of Materials Research 7, 3242-3249 (1992).

9. Y. Xiang, X. Chen, and J. Vlassak, "Plane-strain bulge test for thin films," Journal of materials research 20, 2360-2370 (2005).

10. J. Neggers, J. Hoefnagels, and M. Geers, "On the validity regime of the bulge equations," Journal of materials research 27, 1245-1250 (2012).

11. M. J. Madou, Fundamentals of Microfabrication (CRC Press LLC, Boca Raton, 2002).

12. J. Rogers, T. Someya, and Y. Huang, "Materials and mechanics for stretchable electronics." Science 327, 1603-1607 (2010).

13. K. Han, M. Ciccotti, and S. Roux, "Measuring nanoscale stress intensity factors with an atomic force microscope," Europhysics Letters 89, 1-5 (2010).

14. T. Chu, W. Ranson, and M. Sutton, "Applications of digital-image-correlation techniques to experimental mechanics," Experimental mechanics pp. 232-244 (1985).

15. H. Bruck, S. McNeill, M. Sutton, and W. Peters, "Digital image correlation using Newton-Raphson method of partial differential correction," Experimental Mechanics pp. 261-267 (1989).

16. F. Hild and S. Roux, "Digital Image Correlation: from Displacement Measurement to Identification of Elastic Properties - a Review," Strain 42, 69-80 (2006).

17. F. Hild and S. Roux, "Comparison of Local and Global Approaches to Digital Image Correlation," Experimental Mechanics 52, 1503-1519 (2012).

18. H. Schreier, J. Braasch, and M. Sutton, "Systematic errors in digital image correlation caused by intensity interpolation," Optical Engineering 39, 2915-2921 (2000).

19. H. Schreier and M. Sutton, "Systematic Errors in Digital Image Correlation Due to Undermatched Subset Shape Functions," Experimental Mechanics 42, 303-310 (2002).

20. J. Neggers, J.P.M. Hoefnagels, F. Hild, S. Roux, M.G.D. Geers. A Global Digital Image Correlation Enhanced Full-Field Bulge Test, Procedia IUTAM 4, 73-81 (2012)

21. R. Hill, "A theory of the plastic bulging of a metal diaphragm by lateral pressure," Philosophical Magazine 4, 1133-1142 (1950).

22. F. Hsu, C. Schwab, D. Rigamonti, and J. Humphrey, "Identification of response functions from axisymmetric membrane inflation tests: implications for biomechanics," International Journal of Solids and Structures 31, 3375-3386 (1994).

23. S. Avril, M. Bonnet, A.-S. Bretelle, M. Grédiac, F. Hild, P. Ienny, F. Latourte, D. Lemosse, S. Pagano, E. Pagnacco, and F. Pierron, "Overview of identification methods of mechanical parameters based on full-field measurements," Experimental Mechanics 48, 381-402 (2008).

24. A. Maynadier, M. Poncelet, K. Lavernhe, and S. Roux, "One-shot measurement of thermal and kinematic fields: Infra-red image correlation (iric)," Experimental Mechanics 52, 241-255 (2011). 